\title{
Evaluation of the Dutch BRCA1/2 clinical genetic center referral criteria in an unselected early breast cancer population
}

\author{
Alexandra J van den Broek ${ }^{1,2}$, Karen de Ruiter' ${ }^{1}$, Laura J van 't Veer², Rob AEM Tollenaar'3, \\ Flora E van Leeuwen ${ }^{1}$, Senno Verhoef ${ }^{4}$ and Marjanka K Schmidt ${ }^{\star, 1,2}$
}

In this study, we evaluated the diagnostic value of the Dutch Clinical Genetic Center (CGC) referral guidelines for BRCA1/2 mutation testing in 903 early breast cancer patients, unselected for family history, diagnosed in a cancer hospital before the age of 50 years in 1974-2002; most prevalent Dutch pathogenic BRCA1/2 mutations had been analyzed on coded DNA in a research setting. Forty-nine (5.4\%) of the patients were proven to be BRCA1/2 mutation carriers. We found that $78 \%$ and $69 \%$ of BRCA1 and BRCA2 mutation carriers identified met the criteria for referral to the CGC based on age, family history and synchronous multiple tumors; reflected by a combined sensitivity of $75.5 \%$ and specificity of $63.2 \%$. More than half of the BRCA1 mutation carriers, that is, $58 \%$ had a triple-negative tumor. The highest AUC was obtained by shifting the age at diagnosis threshold criterion from $\mathbf{4 0}$ to 35 years and by adding a 'triple-negative breast cancer' criterion with an age threshold of 45 years; the specificity increased to $71.2 \%$, whereas the sensitivity remained the same; that is, a referral of fewer patients will lead to the identification of at least the same number of BRCA1/2 mutation carriers. Two-thirds of the BRCA1/2 mutation carriers identified in this research setting had been referred for counseling and testing. Our results indicate that, awaiting a possibly more extended mutation screening of all breast cancer patients, the triple-negative status of a breast cancer should be added to the CGC referral criteria.

European Journal of Human Genetics (2015) 23, 588-595; doi:10.1038/ejhg.2014.161; published online 20 August 2014

\section{INTRODUCTION}

The risk of developing breast cancer in BRCA1 and BRCA2 mutation carriers is high, and is estimated to be between 27 and $80 \%$ up to 70 years of age. ${ }^{1-5}$ BRCA1/2 mutation carriers are more likely to develop breast cancer at a young age and have a higher risk of developing a second breast cancer in comparison with women without a mutation in the BRCA1 or BRCA2 gene. ${ }^{2,6}$ Additionally, they also have a high risk of developing ovarian cancer, which is estimated to be between 5 and $60 \%$ up to 70 years of age. ${ }^{1-5}$ Because of the high risks, it is important to identify BRCA1/2 mutation carriers and offer them options to manage their risks; that is, more intensive screening or risk-reducing surgery (prophylactic mastectomy and/or salpingo-oophorectomy). ${ }^{7-10}$ Additionally, it is important to identify breast cancer patients with a BRCA1/2 mutation because of suggestions that targeted chemotherapy will be available for this patient group in the near future; for example, BRCA1/2 mutation carriers have been shown to respond to treatment with PARP inhibitors. ${ }^{10-12}$

The prevalence of women carrying a BRCA1/2 mutation in the general population is low,,$^{13}$ and within breast cancer patients it was estimated to be around $1-2 \%$ for each gene; ${ }^{14}$ testing every breast cancer patient for a mutation is debatable. Except for costeffectiveness and feasibility reasons, there are also considerations regarding the impact of the counseling and testing procedure. ${ }^{15}$ In many Western countries, with no highly prevalent BRCA1/2 founder mutations, the guidelines state that only patients should be tested with an a priori change of at least $10 \%$ of having a mutation. ${ }^{16,17}$ The Clinical Genetic Center (CGC) referral criteria for breast cancer patients are based on family history of the patient and age at diagnosis of breast cancer. ${ }^{16}$ Tumor type, that is, the receptor status of the tumor (estrogen receptor negative $(\mathrm{ER}-)$, progesterone receptor negative $(\mathrm{PR}-)$ and HER2/neu receptor negative (HER2 -)), is suggested to improve the identification of BRCA1 mutation carriers, ${ }^{18,19}$ but is not yet included in most of the CGC referral criteria. ${ }^{16,17,20}$ When a patient is referred to the CGC, DNA testing will be offered when the probability of being a carrier of a $B R C A 1$ and/or BRCA2 mutation is estimated to be over $10 \%$ by the clinical geneticist. ${ }^{21-25}$

In this study, we were able to evaluate the performance of the current Dutch CGC referral criteria $^{17}$ for BRCA1/2 mutation screening, used by clinical geneticists and oncologists in current practice, in an unselected breast cancer patient population diagnosed under the age of 50 years. Changes in sensitivity and specificity were explored by adding the tumor subtype to the criteria and shifting the age at diagnosis thresholds. Furthermore, we studied which part of the BRCA1/2 mutation carriers identified in the research setting had actually been referred to the CGC.

${ }^{1}$ Division of Psychosocial Research and Epidemiology, Netherlands Cancer Institute, Amsterdam, the Netherlands; ${ }^{2}$ Division of Molecular Pathology, Netherlands Cancer Institute, Amsterdam, the Netherlands; ${ }^{3}$ Department of Surgery, Leiden University Medical Center, Leiden, the Netherlands; ${ }^{4}$ Family Cancer Clinic, Netherlands Cancer Institute, Amsterdam, the Netherlands

*Correspondence: Dr MK Schmidt, Division of Psychosocial Research and Epidemiology, Netherlands Cancer Institute, Plesmanlaan 121, Amsterdam 1066 CX, the Netherlands. Tel: +31 (0)20 512 2487; Fax: +31(0)20 512 2322; E-mail: mk.schmidt@nki.nl

Received 28 February 2014; revised 26 June 2014; accepted 9 July 2014; published online 20 August 2014 


\section{METHODS}

\section{Patient selection}

Our study population, a retrospectively ascertained cohort, consists of a consecutive series of invasive early breast cancer patients unselected for a family history of cancer and diagnosed under the age of 50 years. Patients included in the study were treated for primary breast cancer between 1970 and 2003 in the Netherlands Cancer Institute, a cancer hospital in Amsterdam, the Netherlands. Patients with metastases at diagnosis or with earlier tumors were excluded. The breast cancer patients were identified through the Medical Registry of the hospital $(N=1893)$. An overview of the inclusion of patients in the analyses is shown in Figure 1

\section{Germline DNA collection and BRCA1/2 testing (research setting)}

For 1620 breast cancer patients (86\%), we were able to gather germline DNA of sufficient quality and successfully perform BRCA1 and BRCA2 mutation analysis. For most of the patients formalin-fixed, paraffin-embedded tissue blocks containing normal tissue were used for DNA isolation (77\%); for $23 \%$ of the patients, blood was collected instead. The methods for DNA isolation and mutation analysis have been described elsewhere. ${ }^{26}$ In short, DNA was isolated using standard laboratory procedures. BRCA1/2 mutation analysis included testing for 92 pathogenic variants using allelic discrimination or fragment length analyses; sequencing was used for confirmation of mutations. We estimated that with these methods we were able to capture about $61 \%$ of the BRCA1/2 mutations prevalent in families in the Netherlands. ${ }^{26}$ The BRCA1/2 mutations have been submitted to http://databases.lovd.nl/shared/ genes/BRCA1 and http://databases.lovd.nl/shared/genes/BRCA2.

\section{Family history and clinicopathological data collection}

For 903 breast cancer patients included in the cohort, both family history at the time of the breast cancer diagnosis and receptor expression data of the tumor were available. First- and second-degree family history data of the patients at the time of their breast cancer diagnosis, routinely recorded by the treating physician in the patient anamnesis, were collected through the Medical Registry of the hospital. Data regarding the immunohistochemical expression of the ER, PR and HER2 of the breast tumor were gathered from original pathology reports (72\% ER/PR and 53\% of HER2 status) or determined using tissue microarray (28\% ER/PR and 47\% HER2; a tumor was scored negative following the clinically used cutoff points in the Netherlands, i.e., ER $<10 \%$, PR $<10 \%$ and HER2: 0 or $1+) .27,28$ Patients with a tumor without the expression of ER, PR and HER2 were considered to have a triple-negative tumor. The 903 patients with complete available data (56\% of the total cohort) had a similar median age at diagnosis compared with the rest of the cohort (44 vs $43, P$-value $=0.07)$, although the median year of diagnosis was somewhat higher (1994 vs 1991 (range for both: 1974-2002), P-value $\leq 0.001$ )).

\section{Definition of the CGC referral criteria}

The CGC referral criteria used in this study were based on the Dutch situation. ${ }^{17}$ The first criterion is breast cancer diagnosed under the age of 40 years. The second criterion is synchronous bilateral breast cancer (a primary tumor in both breasts) or multiple breast cancer (more primary tumors in one breast); we considered patients with two primary breast tumors diagnosed up to within 3 months apart as synchronous bilateral. The third criterion is when a patient has three or more relatives with cancer, of whom at least one had breast cancer. The family history data available in our hospital medical registry did not completely follow the Dutch CGC referral criteria; ${ }^{17}$ therefore, we slightly adapted the family history criteria for the use in our study (Supplementary Table 1).

\section{Referral of patients to the CGC}

In the 'Progeny' registry database of the CGC of the Netherlands Cancer Institute, information about all patients referred to the CGC is recorded. Through linkage with this database, we identified breast cancer patients from our cohort who were referred to this CGC until April 2012. For these patients, data regarding the date of referral, whether a patient was tested for $B R C A 1 / 2$ mutations in the CGC and the testing results, were collected from Progeny. Also, full pedigree family history data, recorded by the clinical geneticist for all referred patients and updated at every visit to the CGC by the patient and/or any relatives, were available.

\section{Coded data linkage}

Using a coding procedure, ${ }^{29}$ the clinical data and $B R C A 1 / 2$ mutation study results were anonymized before linkage. As discussed earlier, ${ }^{29}$ no explicit

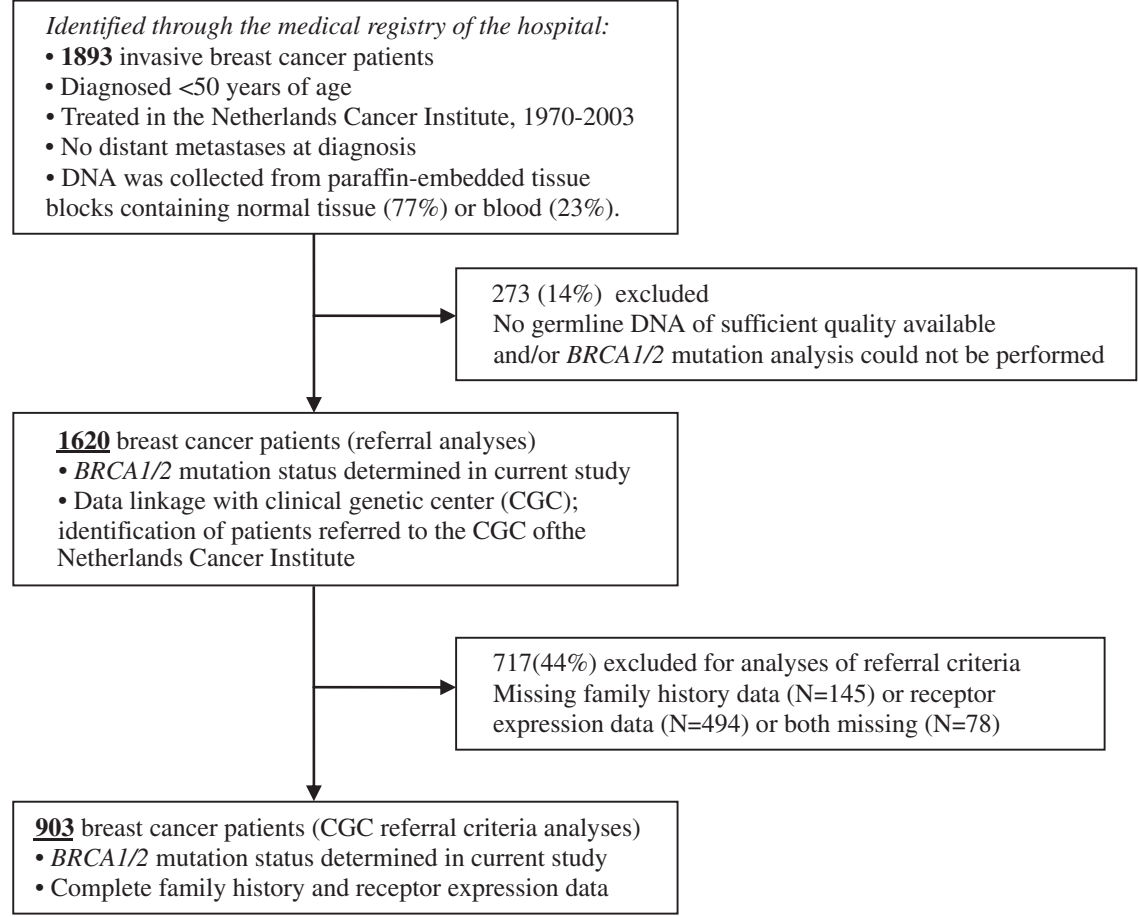

Figure 1 Inclusion of patients in the study and in the analyses. 
informed consent was asked and results were not reported back to the patients. The secondary use of long-term stored tissue samples and clinical data in this study was according to Dutch guidelines (Dutch codes of conduct: http:// www.federa.org/codes-conduct) and approved by the review board of the Netherlands Cancer Institute.

\section{Statistical analyses}

Differences in proportions of breast cancer patients with a family history and other clinicopathological characteristics were tested for an association with the $B R C A 1$ and BRCA2 mutation status, as determined in our research cohort, using the Pearson's $\chi^{2}$-test.

Analyses of the performance of the CGC referral criteria to identify women who proved to be BRCA1/2 mutation carriers in our research cohort. The sensitivity, specificity, positive-predictive and negative-predictive values were calculated for the CGC referral criteria to identify the BRCA1/2 mutation carriers in our research cohort (for the used methods see Tables 2 and 3). The discriminative performance between $B R C A 1 / 2$ mutation carriers and non-carriers was measured by the area under the receiver-operating characteristic curve (AUC) of the logistic regression model including the CGC referral criteria. Changes in sensitivity and specificity were explored by shifting the threshold of the age at diagnosis of breast cancer and by adding and shifting the threshold of the age at diagnosis of triple-negative breast cancer; the criteria regarding family history and bilateral/multiple breast cancer were kept unchanged. The AUC of the models including criteria with shifted thresholds were compared with the AUC of the original model including the CGC referral criteria using a $\chi^{2}$-test. A sensitivity analysis was performed by making the family history criterion less specific: all breast cancer cases with at least one family member with breast cancer at the time of diagnosis were taken into account instead of only cases with more than two relatives with breast and/or other cancer (at least one must have had breast cancer). Statistical analyses were performed using STATA (STATA version 11.2; STATA Corporation, College Station, TX, USA).

Analyses of the CGC referral patterns of women who proved to be BRCA1/2 mutation carriers in our research cohort. Proportions of patients who were referred and were not referred, related to the BRCA1/2 mutation status as determined in our research cohort, were determined and patient characteristics (age at diagnosis of the tumor; family history) were summarized for the different groups. All the above analyses were stratified for calendar year of diagnosis of breast cancer: $<1995$ compared with >1994; the year of the discovery of the BRCA1/2 genes and the start of testing in the Netherlands. Furthermore, for patients diagnosed after 1994, time of referral was used to distinguish patients who were referred to the CGC of the Netherlands Cancer Institute before or within 1 year after their breast cancer diagnosis, and those who were referred more than 1 year after their breast cancer diagnosis or were not referred (including 17 (13\%) patients with an unknown referral date).

\section{RESULTS}

Forty-nine $(5.4 \%)$ women were proven to be BRCA1/2 mutation carriers in our research cohort of 903 breast cancer patients diagnosed under the age of 50 years. Characteristics of the BRCA1 mutation carriers, $B R C A 2$ mutation carriers and non-carriers in our cohort are shown in Table $1 ; 80.6 \%$ and $76.7 \%$ of BRCA1 and BRCA2 mutation carriers, respectively, had one or more family members with breast cancer at the time of breast cancer diagnosis compared with $46.0 \%$ of the non-carriers. In all, $58.3 \%$ of the BRCA1 mutation carriers had a triple-negative tumor compared with only $13.2 \%$ and $7.7 \%$ of the non-carriers and BRCA2 mutation carriers, respectively.

Performance of the CGC referral criteria to identify women who proved to be BRCA1/2 mutation carriers in our research cohort. Each CGC referral criterion, except the bilateral/multiple breast cancer criterion that only included small numbers, identified more than $40 \%$ of the women who proved to be $B R C A 1$ or $B R C A 2$ mutation carriers in our research cohort (Table 1).

In all, $77.8 \%$ of the breast cancer patients with a $B R C A 1$ and $69.2 \%$ of the breast cancer patients with a BRCA2 mutation fulfilled at least one of the CGC referral criteria, compared with $36.8 \%$ of the non-carriers. No patient in our cohort met all the criteria (Table 1).

The sensitivity and specificity of the CGC referral criteria to identify the BRCA1/2 mutation carriers in our research cohort were $75.5 \%$ and $63.2 \%$, respectively (Table 2). The corresponding AUC was 0.73 (Supplementary Figure 1). The sensitivity of each individual criterion was poor, whereas specificity was high (Table 2). The sensitivity and specificity of the CGC referral criteria to identify only the BRCA1 mutation carriers were $77.8 \%$ and $62.7 \%$, respectively (Supplementary Table 2).

In three different settings in which the triple-negative criterion was added to the CGC referral criteria and the age thresholds in the criteria shifted, the absolute increase of the specificity was up to $6.8 \%$, whereas the sensitivity was equal or also increased (2.1\%), compared with the original CGC referral criteria (Table 3). Accordingly, there was an increase in the AUC of 0.04 and 0.05 when the age at diagnosis threshold of all breast cancer cases was decreased to 35 years and the age at diagnosis threshold of the triple-negative breast cancer cases was added and set at 50 years (Supplementary Figure 1A; $P$-value $=0.21$ ) or 45 years (Supplementary Figure $1 \mathrm{~B} ; \quad P$ value $=0.08$ ), respectively. In the last setting, with the highest increase in discriminative performance compared with the original CGC referral criteria, only three extra mutation carriers were identified in addition to those identified by the original CGC referral criteria (Supplementary Figure 2). However, fewer non-carriers met the shifted criteria than the original CGC referral criteria ( $N$ difference $=68$; Supplementary Figure 2). In other words, less patients will have to be tested to identify the same number of BRCA1/ 2 mutation carriers, explaining the improved specificity and overall discriminative performance.

Referring to the Dutch NABON guidelines, under patients who are considered for referral, a 10\% level of detection rate of BRCA1/2 mutation carriers is desired. ${ }^{17}$ In our research cohort, the percentage of $B R C A 1 / 2$ mutation carriers under patients who fulfilled the family history criterion and/or the bilateral/ multiple breast cancer criterion was $13.7 \%$ (Supplementary Table 3A). Among patients who did not fulfill these criteria, only in patients diagnosed with breast cancer under the age of 30 years, the desired level of $10 \%$ detection rate of mutation carriers was reached. The proportion of BRCA1/2 mutation carriers among patients with a triple-negative tumor was higher than $10 \%$ in all age groups except in the 45-50 years age groups (Supplementary Table 3A).

In the sensitivity analyses in which the family history criterion was made less stringent, that is, all women with at least one relative with breast cancer were considered to fulfill the criterion, overall the results did not change and confirmed the original results, although the effects found were less pronounced (Supplementary Tables 3B and 4).

The CGC referral patterns of women who proved to be BRCA1/2 mutation carriers in our research cohort $(N=1620)$. Of the breast cancer patients included in our cohort and diagnosed before 1995, that is, the discovery of the BRCA1/2 genes $(<1995), 14 \%$ had been referred to the CGC of the Netherlands Cancer Institute by April 2012 (Figure 2a). Woman who were proven to be BRCA1/2 mutation 
Table 1 Distribution of clinicopathological characteristics and the CGC referral criteriaa,b

\begin{tabular}{|c|c|c|c|c|c|c|c|c|}
\hline \multirow[b]{2}{*}{ BRCA1/2 mutation status ${ }^{c}$} & \multicolumn{2}{|c|}{ Non-carriers } & \multicolumn{2}{|c|}{ BRCA1 } & \multirow[b]{2}{*}{$P$-value ${ }^{\mathrm{d}}$} & \multicolumn{2}{|c|}{ BRCA2 } & \multirow[b]{2}{*}{$P$-value } \\
\hline & $N^{e}$ & $\%$ & $N^{e}$ & $\%$ & & $N^{e}$ & $\%$ & \\
\hline Total $N=903$ & 854 & 94.6 & 36 & 4.0 & & 13 & 1.4 & \\
\hline Median age diagnosis (years, SD) & 44 & 5.4 & 36 & 7.6 & $<0.001$ & 39 & 4.5 & 0.002 \\
\hline Bilateral/multiple breast cancer & 6 & 0.7 & 2 & 5.6 & 0.003 & 0 & 0 & NA \\
\hline Triple-negative tumor & 113 & 13.2 & 21 & 58.3 & $<0.001$ & 1 & 7.7 & 0.56 \\
\hline \multicolumn{9}{|l|}{ Family history (type ${ }^{f}$ ) } \\
\hline No affected relatives & 310 & 36.3 & 1 & 2.8 & $<0.001$ & 1 & 7.7 & 0.06 \\
\hline Yes, breast cancer & 393 & 46.0 & 29 & 80.6 & & 10 & 76.9 & \\
\hline Yes, only other cancers & 151 & 17.7 & 6 & 16.7 & & 2 & 15.4 & \\
\hline \multicolumn{9}{|l|}{ Family history (numberg) } \\
\hline No affected relatives & 310 & 36.3 & 1 & 2.8 & $<0.001$ & 1 & 7.7 & 0.006 \\
\hline Yes, $<3$ & 373 & 43.7 & 18 & 50.0 & & 5 & 38.5 & \\
\hline Yes, $\geq 3$ & 171 & 20.0 & 17 & 47.2 & & 7 & 53.9 & \\
\hline \multicolumn{9}{|l|}{ CGC referral criteria ${ }^{a}$} \\
\hline (A) Three or more family members with cancer ${ }^{\mathrm{h}}$ & 146 & 17.1 & 16 & 44.4 & $<0.001$ & 6 & 46.2 & 0.006 \\
\hline (B) Bilateral/multiple breast cancer & 6 & 0.7 & 2 & 5.6 & 0.003 & 0 & 0 & NA \\
\hline (C) Breast cancer diagnosed $<40$ years & 209 & 24.5 & 21 & 58.3 & $<0.001$ & 7 & 53.8 & 0.02 \\
\hline At least one criterion met & 314 & 36.8 & 28 & 77.8 & $<0.001$ & 9 & 69.2 & 0.02 \\
\hline \multicolumn{9}{|l|}{ Number of BRCA1/2 screening criteria met } \\
\hline 1 & 267 & 85.0 & 17 & 60.7 & 0.001 & 5 & 55.6 & 0.02 \\
\hline 2 & 47 & 15.0 & 11 & 39.3 & & 4 & 44.4 & \\
\hline 3 & 0 & 0 & 0 & 0 & & 0 & 0 & \\
\hline \multicolumn{9}{|c|}{ Proportions BRCA1/2 mutation carriers under all patients, by age diagnosis (years) } \\
\hline$<35$ & 75 & 81.5 & 15 & 16.3 & $<0.001$ & 2 & 2.2 & 0.02 \\
\hline $35-40$ & 134 & 92.4 & 6 & 4.1 & & 5 & 3.5 & \\
\hline $40-45$ & 242 & 94.5 & 9 & 3.5 & & 5 & 2.0 & \\
\hline $45-50$ & 403 & 98.3 & 6 & 1.5 & & 1 & 0.2 & \\
\hline \multicolumn{9}{|c|}{ Proportions BRCA1/2 mutation carriers under patients with triple-negative tumors, by age diagnosis (years) } \\
\hline$<35$ & 19 & 61.3 & 12 & 38.7 & $<0.001$ & 0 & 0 & 0.12 \\
\hline $35-40$ & 16 & 80.0 & 3 & 15.0 & & 1 & 5.0 & \\
\hline $40-45$ & 25 & 86.2 & 4 & 13.8 & & 0 & 0 & \\
\hline $45-50$ & 53 & 96.4 & 2 & 3.6 & & 0 & 0 & \\
\hline
\end{tabular}

Abbreviations: CGC, clinical genetic centre; NA, not applicable.

${ }^{\text {aAdjusted criteria as described in Supplementary Table } 1 .}$

bAnalyses among included breast cancer patients stratified by the BRCA1/2 mutation status as determined in our research cohort.

${ }^{C}$ As determined in this research setting.

d $P$-value of the Pearson's $\chi^{2}$-test, BRCA1 or BRCA2 carriers compared with non-carriers.

eUnless otherwise specified.

fType of cancer present in affected relatives.
Types otherwise specified.

Type of cancer present in affected relatives.
gNumber of affected relatives in the family.

hAt least one must have had breast cancer.

carriers in our research cohort were more frequently referred compared with the non-carriers in our cohort $(34 \%$ vs $13 \%, P$-value $<0.001)$. The referral time ranged from 4 months to more than 29 years after the breast cancer diagnosis (range referral years: 1995-2011).

Fifteen percent of all breast cancer patients included in our cohort and diagnosed after 1994 had been referred to the CGC of the Netherlands Cancer Institute before $(2 \%)$ or within 1 year after diagnosis (14\%) (Figure 2b). Patients who were referred before diagnosis $(N=12)$ all had a family history of breast cancer at the time of diagnosis; most of them $(N=11)$ even had three or more relatives affected with cancer. Furthermore, patients referred not before but within 1 year after diagnosis were more likely to have a family history of breast cancer at the time of diagnosis ( $86 \%$ vs $44 \%$, $P$-value $<0.001)$, to have more affected relatives with cancer $(35 \% v s$ $20 \%, P$-value $=0.001)$ and to be diagnosed with breast cancer under the age of 35 years $(32 \%$ vs $9 \%, P$-value $<0.001)$ than patients who were not referred to the CGC of the Netherlands Cancer Institute before or within a year after breast cancer diagnosis. However, of these patients who were not referred, $17 \%$ still had an extensive family history at the time of diagnosis (three or more relatives with cancer, of whom at least one had breast cancer) (Figure 2b).

Under the patients diagnosed with breast cancer after 1994, the proportion who had been referred to the CGC of the Netherlands Cancer Institute before diagnosis was higher among BRCA1/2 mutation carriers than among the non-carriers identified in our research cohort $(15 \%$ vs $1 \%, P$-value $<0.001)$. Also, the proportion of patients who were referred not before but within 1 year after diagnosis was higher among the carriers $(26 \%$ vs $13 \%, P$-value $<0.001)$ (Figure $2 \mathrm{~b}$ ). These referred carriers all had a family history of breast cancer, of whom eight (50\%) had more than three affected relatives with cancer, compared with $74 \%$ of the BRCA1/2 mutation carriers who were not referred before or within 1 year after diagnosis $(P$ - 
Table 2 Performance of each individual criterion and the CGC referral criteriaa,b

\begin{tabular}{|c|c|c|c|c|c|c|c|c|c|}
\hline & $\begin{array}{c}\text { No. } \\
\text { falsenegatives }\end{array}$ & $\begin{array}{l}\text { No. true } \\
\text { negatives }\end{array}$ & $\begin{array}{l}\text { No. false } \\
\text { positives }\end{array}$ & $\begin{array}{l}\text { No. true } \\
\text { positives }\end{array}$ & & & & & \\
\hline & & & & & & $\%$ of $B R C A 1 / 2$ & & & \\
\hline Criterion met: & No & No & Yes & Yes & the criterion & value $e^{c}$ & value & Sensitivity & Specificity \\
\hline (C) Breast cancer diagnosed $<40$ years & 21 & 645 & 209 & 28 & 237 & 11.8 & 96.8 & 57.1 & 75.5 \\
\hline CGC referral criteria ${ }^{a}$ - all above criteria combined & 12 & 540 & 314 & 37 & 351 & 10.5 & 97.8 & 75.5 & 63.2 \\
\hline Triple-negative breast cancer (no age limitation) & 27 & 741 & 113 & 22 & 135 & 16.3 & 96.5 & 44.9 & 86.8 \\
\hline
\end{tabular}

Table 3 Performance of adaptions of the CGC referral criteria ${ }^{a, b}$

Criteria shift

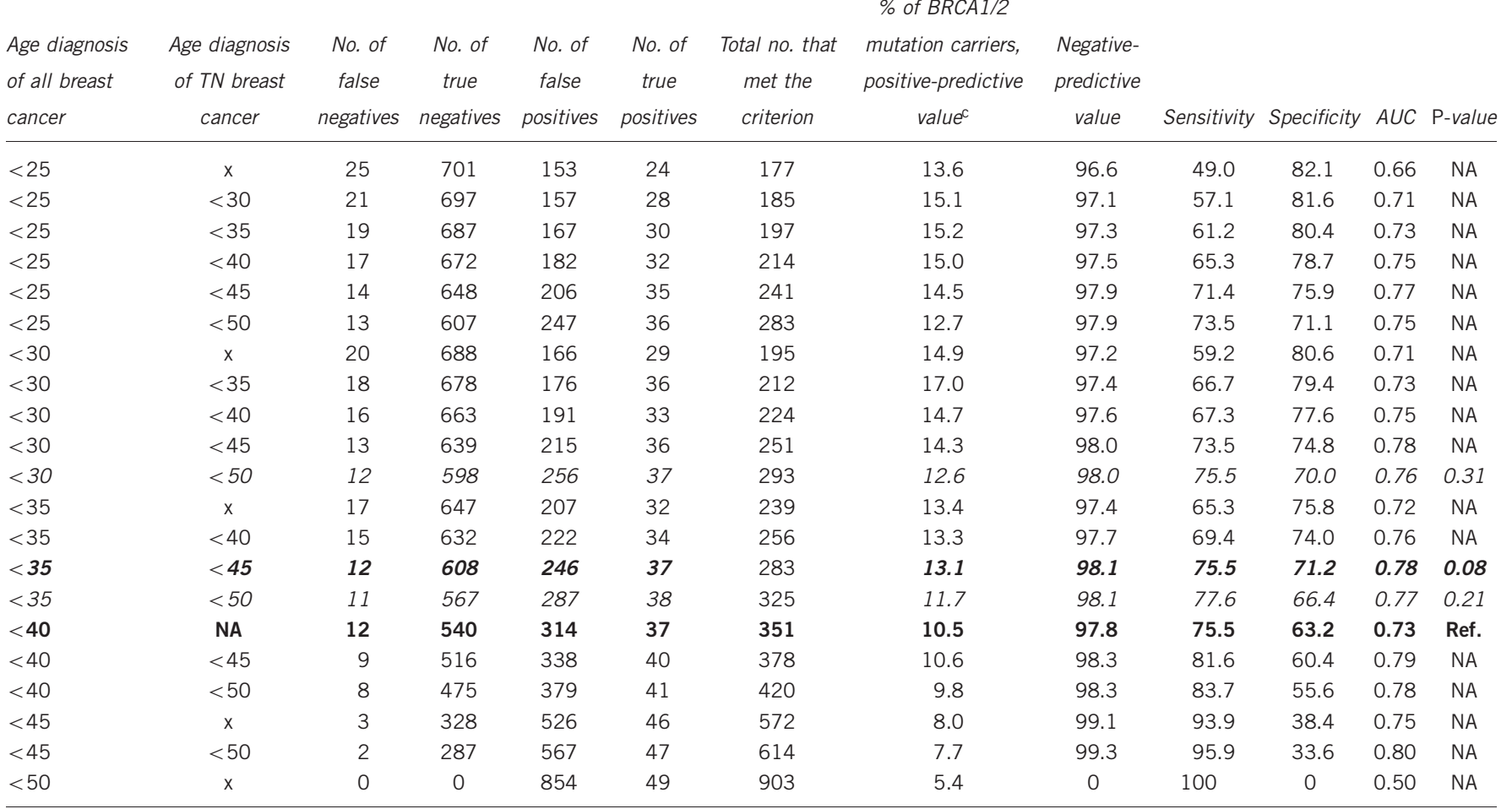

Abbreviations: NA, not applicable; negative-predictive value, no. of non-carriers that did not meet the CGC referral criteria/total no. that did not meet the CGC referral criteria; positive-predictive value, no. of BRCA mutation carriers that met the CGC referral criteria/total no. that met the CGC referral criteria; sensitivity, no. of BRCA mutation carriers that met the CGC referral criteria/total value, no. of $B R C A$ mutation carriers that met the CGC referral criteria/total no. that met the CGC referral criteria; sensitivity, no. of BRCA mutation carriers that
no. of $B R C A$ mutation carriers; specificity, no. of non-carriers that did not meet the CGC referral criteria/total no. of non-carriers; $\mathrm{x}$, no change in the criterion..

${ }^{a}$ Adjusted criteria (based on the Dutch situation) as described in Supplementary Table 1; bold represents the CGC referral criteriaa; italics represents the adjusted criteria achieving an equal or higher sensitivity and higher specificity than the CGC referral criteriaa ; bold and italics represent the adjusted criteria achieving an equal or higher sensitivity and higher specificity than the CGC referral criteria, and with the best discriminatory performance (highest AUC).

${ }^{b}$ Analyses to identify women who proved to be BRCA1/2 mutation carriers in our research cohort when shifting the age at diagnosis of breast cancer and shifting the age at diagnosis of triplenegative breast cancer. No changes in the other criteria were made.

${ }^{c}$ Diagnostic yield: Prior chance on detecting a mutation.

value $=0.03$; Figure $2 \mathrm{a})$. Furthermore, nine $(56 \%)$ of the referred $B R C A 1 / 2$ mutation carriers were diagnosed with breast cancer at a very young age (under 35 years) compared with six $(26 \%)$ of the carriers who were not referred before or within 1 year after their diagnosis $(P$-value $=0.06)$.
Ten additional BRCA1/2 mutation carriers diagnosed after 1994 were referred to the CGC in the later years after diagnosis. Eventually, $67 \%(N=26$, taking into account 2 with an unknown referral date) of the total group of women who proved to be $B R C A 1 / 2$ carriers in our research cohort were referred and were 
a
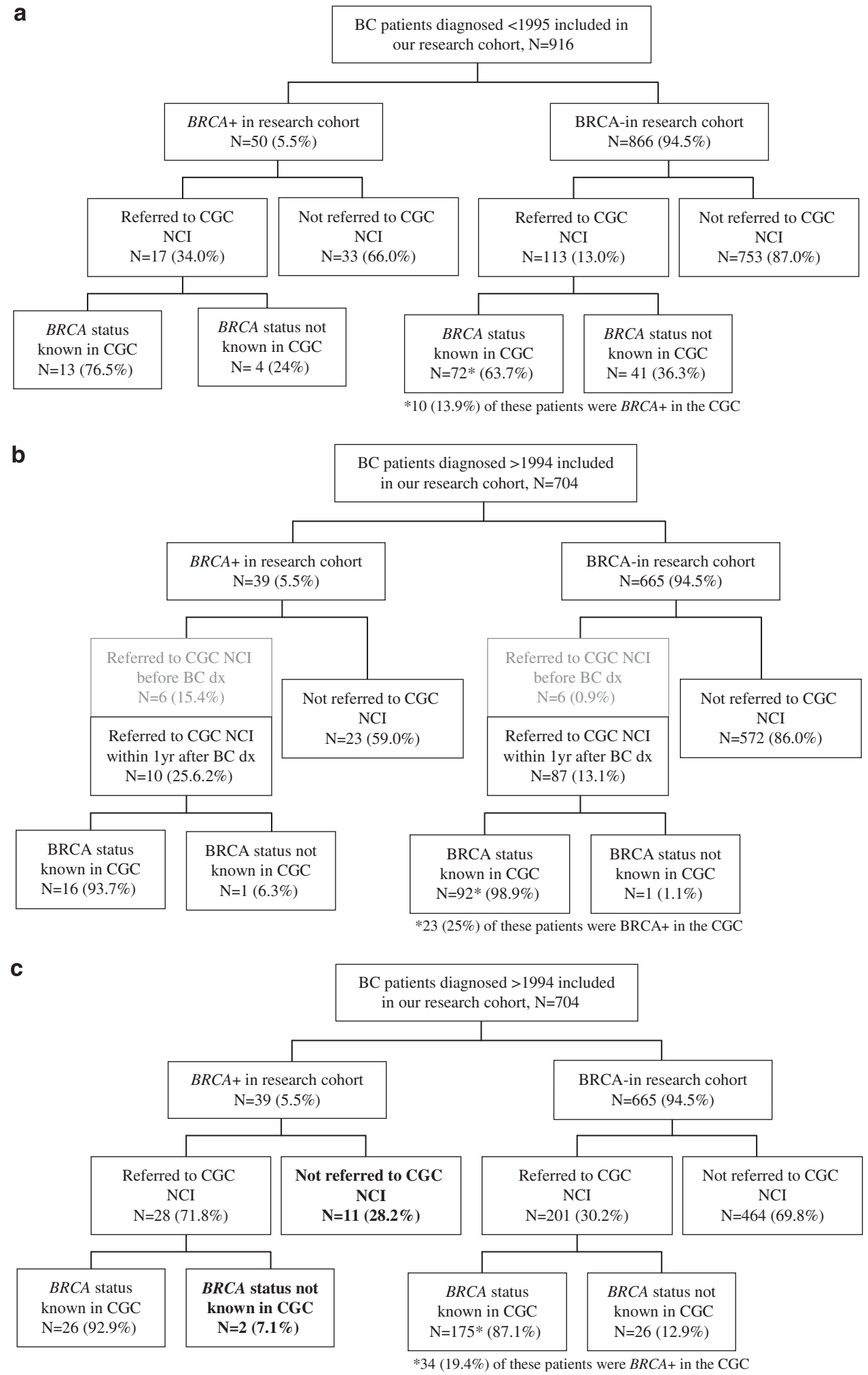

Figure 2 Overview of the women who proved to be BRCA1/2 mutation carriers and non-carriers in our research cohort and referral of these patients to the CGC of the Netherlands Cancer Institute $(\mathrm{NCl})(N=1620)$, stratified for the calendar year of diagnosis $(\mathrm{a}-\mathrm{C})$. Bold represents the number of patients who are carriers of a BRCA1/2 mutation and were diagnosed after 1994, but who were not referred to the CGC of the NCl or the $B R C A$ status was not known in the CGC of the $\mathrm{NCl}$; BRCA -, BRCA mutation non-carrier as determined in our research cohort; BRCA +, BRCA1/2 mutation carrier as determined in our research cohort. For $166(18 \%)$ patients in panel a family history data was missing; for $57(8 \%)$ patients in panels b and c family history data was missing. (a) Patients diagnosed <1995: all patients referred to the CGC by April 2012 are indicated as referred. (b) Patients diagnosed > 1994: only the patients referred to the CGC before breast cancer diagnosis (gray), or within 1 year after their breast cancer diagnosis are indicated as referred (patients with unknown referral date $(n=17$; of which two BRCA + ) are included in the group 'not referred'). (c) Patients diagnosed > 1994: all patients referred to the CGC by April 2012 are indicated as referred. 
tested for $B R C A 1 / 2$ mutations in the CGC of the Netherlands Cancer Institute (Figure 2c). Thirteen BRCA1/2 mutation carriers had not been referred or were not tested for $B R C A 1 / 2$ mutations by April 2012 (Figure 2c). Four of these carriers were diagnosed in 1995 or 1996, shortly after the discovery of the BRCA1/2 genes; two died shortly ( 1 and 4 years) after the breast cancer diagnosis. Of the seven other carriers, only two had more than two family members with breast cancer at the time of diagnosis (Supplementary Table 5).

\section{DISCUSSION}

Our study is one of the first studies to evaluate the performance of the CGC referral criteria to identify women who proved to be BRCA1/2 mutation carriers in an unselected hospital-based cohort of breast cancer patients. Most earlier publications evaluated risk assessment models, which are based on extensive pedigree information and are most suitable after a patient has been referred to the CGC. ${ }^{30} \mathrm{We}$ calculated that the CGC referral criteria (based on the Dutch situation) had a sensitivity of $75.5 \%$ and specificity of $63.2 \%$ to identify the $B R C A 1 / 2$ mutation carriers. In the group of patients in our cohort who met the CGC referral criteria $(N=351)$, there was a detection rate for $B R C A 1 / 2$ mutation carriers of $10.5 \%$. An improved performance to identify $B R C A 1 / 2$ mutation carriers, that is, a better specificity (up to $6.8 \%$ absolute increase) and similar or better sensitivity (up to $2.1 \%$ increase), and accordingly an increase in detection rate up to $13.1 \%$, was achieved by shifting the threshold of the age at diagnosis of breast cancer from 40 to 35 years and by adding an age threshold to 45 or 50 year for patients with a triple-negative breast cancer. In conclusion, to identify the same number or even more BRCA1/2 mutation carriers, fewer patients need to be referred to the CGC.

It should be noted here that our results are most important for referral of $B R C A 1$ mutation carriers: they are more often diagnosed with triple-negative tumors compared with BRCA2 mutation carriers (Table 1). ${ }^{31}$ When performing our analyses on BRCA1 mutation carriers only, we saw stronger and significant effects of the addition of triple-negative status to the CGC referral criteria on the sensitivity and specificity (Supplementary Table 2). We considered there was insufficient power to perform the analyses separately for $B R C A 2$ mutation carriers. Moreover, in clinical practice mutation testing is mostly performed for both genes simultaneously, and thus separation of the results is less relevant.

When interpreting the results from this study, it should be taken into account that our study is performed in a research setting; we estimated that we were able to screen for about $61 \%$ of the pathogenic BRCA1/2 mutations (69\% BRCA1 mutations; 46\% BRCA2 mutations) known to be prevalent in Dutch families. ${ }^{26}$ We screened for a higher percentage of BRCA1 mutations than BRCA2 mutations, and $B R C A 2$ mutation carriers are less prevalent in the Dutch population. ${ }^{26}$ Consequently, we missed BRCA1/2 mutation carriers in our cohort (see Figure 2) and we may assume results reported here are conservative and most important for the referral of BRCA1 mutation carriers. In addition, it should be kept in mind that the difference in absolute numbers of $B R C A 1 / 2$ mutation carriers comparing the original and altered CGC referral criteria was small.

The earlier CGC referral criteria were solely based on the patients' family history of cancer. ${ }^{32}$ In line with this, we noticed that BRCA1/2 mutation carriers identified in our research cohort, who were diagnosed with breast cancer after 1994 and referred before or within 1 year after diagnosis, more often had a family history of breast cancer at the time of diagnosis compared with those who were referred later or not at all. Likely, the later referred patient developed another cancer or had additional family members developing cancer Thirteen (33\%) BRCA1/2 mutation carriers identified in our research cohort and diagnosed with breast cancer after 1994 were not referred to the CGC of the Netherlands Cancer Institute by April 2012. Most of them were not referred for logical reasons (these patients were diagnosed shortly after the discovery of the BRCA1/2 genes or died shortly after the breast cancer diagnosis; Supplementary Table 5); in addition, a few, even though with a positive family history of breast cancer at the time of diagnosis, might not have wanted to be referred, ${ }^{33}$ their treating physician lacked knowledge and/or awareness, ${ }^{34}$ or even though diagnosed and treated at The Netherlands Cancer Institute, a specialized cancer center, were screened in another CGC.

The family history of a patient is still the most important criterion for referral of patients to the $\mathrm{CGC}^{17}$ with the highest sensitivity to identify BRCA1/2 mutation carriers (Tables 1 and 2). ${ }^{35}$ Of note, noncarriers with an extensive family history of (breast) cancer are at increased risk of developing second cancers ${ }^{36}$ and referral to the CGC of these patients remains important. As we did not have full pedigree information at the time of diagnosis available in our cohort, we probably missed information of some brothers/fathers affected by breast cancer or family members affected by ovarian cancer; however, we had information also about breast cancer patients non-referred to the CGC. It is difficult to predict what would be the effect of this on the results observed in this study, but in previously published Dutch data, the prevalence of breast cancer patients with ovarian cancer in the family was only $2 \% .{ }^{37}$ That same study reported $34 \%$ of breast cancer patients to have a family history of breast cancer to the extent of a CGC referral indication; ${ }^{37}$ higher than the $19 \%$ in our study, this difference likely being explained by our younger population. Considering these figures, we can postulate that in our cohort the effect of the family history criterion might be somewhat underestimated. However, in a sensitivity analysis with a less specific family history criterion $(48 \%$; taking all breast cancer cases with at least one relative with breast cancer into consideration), we confirmed that decreasing the age at diagnosis threshold of all breast cancer cases and increasing the age at diagnosis threshold of the triple-negative breast cancer cases improves both the sensitivity and specificity of the criteria (Supplementary Table 4).

We also tried to get insight into the quality of the family history data we used in this study, by comparing family history information from the clinical report with the more extended family history data available from the subgroup of CGC referred patients. Not surprisingly, the former data reported fewer patients with a family history of cancer, although this difference was most pronounced for non-breast cancer (data not shown). The CGC data reflected for many patients the status after their diagnosis of breast cancer, or later since pedigrees are updated at every visit to the CGC by the patient and/or any relatives. Since for breast cancer patients it is mostly the treating physician who refers to the CGC, we feel that the data used in our analyses are more representative for the everyday situation in clinical practice. Although this family history information may not have been recorded consistently over the whole study period by clinicians, carrier status was unknown at diagnosis, and therefore it is unlikely that this led to differential bias.

Our results, certainly when taking into account previously published data, ${ }^{16,18,19,38-40}$ indicate that histologic parameters, that is, the triple-negative status of the breast cancer, should be incorporated in the CGC referral criteria. Even though we evaluated specifically the Dutch guidelines, other Western countries are in a similar situation; of seven international guidelines, only two indicate triple-negative breast cancer as an indication for BRCA1/2 testing. ${ }^{20}$ Families in Western countries are becoming smaller and other factors to identify 
$B R C A 1 / 2$ mutation carriers, such as the type of breast tumor, are becoming more important. ${ }^{41}$ It has repeatedly been suggested that pathologic data should be incorporated in addition to family history in the prediction of the probability of carrying a mutation in one of the BRCA1/2 genes. ${ }^{18,38}$ Recent publications show that even testing all women younger than 50 years with triple-negative breast cancer, regardless of family history, is a cost-effective strategy and could reduce subsequent cancer risks, ${ }^{39,40}$ and the NICE guidelines (UK) recently included the triple-negative criterion (<age 40 years). ${ }^{42}$ Expanding the CGC referral criteria with this information will improve cancer risk management, for example, decisions of salpingo-oophorectomy or preventive contralateral surgery and/or treatment with PARP inhibitors, for these women and their families.

\section{CONFLICT OF INTEREST}

The authors declare no conflict of interest.

\section{ACKNOWLEDGEMENTS}

This work was supported by the Dutch Cancer Society grant numbers DCS-NKI 2001-2423, DCS-NKI 2007-3839 and DCS-NKI 2009-4363, and by notary office Spier \& Hazenberg regarding the coding procedure.

1 Antoniou A, Pharoah PD, Narod S et al: Average risks of breast and ovarian cancer associated with BRCA1 or BRCA2 mutations detected in case series unselected for family history: a combined analysis of 22 studies. Am J Hum Genet 2003; 72: 1117-1130.

2 Mavaddat N, Peock S, Frost D et al: Cancer risks for BRCA1 and BRCA2 mutation carriers: results from prospective analysis of EMBRACE 4. J Natl Cancer Inst 2013; 105: 812-822.

3 Brohet RM, Velthuizen ME, Hogervorst FB et al: Breast and ovarian cancer risks in a large series of clinically ascertained families with a high proportion of BRCA1 and BRCA2 Dutch founder mutations 1. J Med Genet 2014; 51: 98-107.

4 King MC, Marks JH, Mandell JB: Breast and ovarian cancer risks due to inherited mutations in BRCA1 and BRCA2. Science 2003; 302: 643-646.

5 Chen S, Parmigiani G: Meta-analysis of BRCA1 and BRCA2 penetrance. J Clin Oncol 2007; 25: 1329-1333

6 Liebens FP, Carly B, Pastijn A, Rozenberg S: Management of BRCA1/2 associated breast cancer: a systematic qualitative review of the state of knowledge in 2006. Eur J Cancer 2007; 43: 238-257.

7 Bermejo-Perez MJ, Marquez-Calderon S, Llanos-Mendez A: Effectiveness of preventive interventions in BRCA1/2 gene mutation carriers: a systematic review. Int J Cancer 2007; 121: 225-231.

8 Domchek SM, Rebbeck TR: Preventive surgery is associated with reduced cancer risk and mortality in women with BRCA1 and BRCA2 mutations. LDI Issue Brief 2010; 16: 1-4.

9 Rebbeck TR, Kauff ND, Domchek SM: Meta-analysis of risk reduction estimates associated with risk-reducing salpingo-oophorectomy in BRCA1 or BRCA2 mutation carriers. J Natl Cancer Inst 2009; 101: 80-87.

10 Narod SA: BRCA mutations in the management of breast cancer: the state of the art. Nat Rev Clin Oncol 2010; 7: 702-707.

11 Sandhu SK, Yap TA, de Bono JS: The emerging role of poly(ADP-ribose) polymerase inhibitors in cancer treatment. Curr Drug Targets 2011; 12: 2034-2044.

12 Sandhu SK, Yap TA, de Bono JS: Poly(ADP-ribose) polymerase inhibitors in cancer treatment: a clinical perspective. Eur J Cancer 2010; 46: 9-20.

13 Rahman N, Stratton MR: The genetics of breast cancer susceptibility. Annu Rev Genet 1998; 32: 95-121.

14 Thompson D, Easton D: The genetic epidemiology of breast cancer genes. J Mammary Gland Biol Neoplasia 2004; 9: 221-236.

15 Surbone A: Social and ethical implications of BRCA testing. Ann Oncol 2011; 22(Suppl 1): i60-i66.

16 Gadzicki D, Evans DG, Harris $\mathrm{H}$ et al: Genetic testing for familial/hereditary breast cancer-comparison of guidelines and recommendations from the UK, France, the Netherlands and Germany. J Community Genet 2011; 2: 53-69.

17 NABON. CBO (Dutch Institute for Healthcare Improvement) guideline for breast cancer; 2012. Available at: http://www.oncoline.nl/mammacarcinoom (last accessed on 26 June 2014)

18 Evans DG, Lalloo F, Cramer A et al: Addition of pathology and biomarker information significantly improves the performance of the Manchester scoring system for BRCA1 and BRCA2 testing. J Med Genet 2009; 46: 811-817.
19 Young SR, Pilarski RT, Donenberg T et al: The prevalence of BRCA1 mutations among young women with triple-negative breast cancer. BMC Cancer 2009; 9: 86.

20 Sharma P, Klemp JR, Kimler BF et al: Germline BRCA mutation evaluation in a prospective triple-negative breast cancer registry: implications for hereditary breast and/ or ovarian cancer syndrome testing 3. Breast Cancer Res Treat 2014; 145: 707-714.

21 Kang HH, Williams R, Leary J, Ringland C, Kirk J, Ward R: Evaluation of models to predict BRCA germline mutations. Br J Cancer 2006; 95: 914-920.

22 Couch FJ, DeShano ML, Blackwood MA et al: BRCA1 mutations in women attending clinics that evaluate the risk of breast cancer. N Engl J Med 1997; 336: 1409-1415.

23 Frank TS, Deffenbaugh AM, Reid JE et al: Clinical characteristics of individuals with germline mutations in BRCA1 and BRCA2: analysis of 10,000 individuals. J Clin Oncol 2002; 20: 1480-1490.

24 Parmigiani G, Berry D, Aguilar O: Determining carrier probabilities for breast cancersusceptibility genes BRCA1 and BRCA2. Am J Hum Genet 1998; 62: 145-158.

25 Evans DG, Eccles DM, Rahman $N$ et al: A new scoring system for the chances of identifying a BRCA1/2 mutation outperforms existing models including BRCAPRO. J Med Genet 2004; 41: 474-480.

26 Schmidt MK et al: BRCA1 and BRCA2 mutation spectrum in a hospital-based series of young breast cancer patients (unpublished).

27 Harvey JM, Clark GM, Osborne CK, Allred DC: Estrogen receptor status by immunohistochemistry is superior to the ligand-binding assay for predicting response to adjuvant endocrine therapy in breast cancer. J Clin Oncol 1999; 17: 1474-1481.

28 Allred DC, Harvey JM, Berardo M, Clark GM: Prognostic and predictive factors in breast cancer by immunohistochemical analysis. Mod Pathol 1998; 11: 155-168.

29 Schmidt MK, Vermeulen E, Tollenaar RA, Van't Veer LJ, van Leeuwen FE: Regulatory aspects of genetic research with residual human tissue: effective and efficient data coding. Eur J Cancer 2009; 45: 2376-2382.

30 Amir E, Freedman OC, Seruga B, Evans DG: Assessing women at high risk of breast cancer: a review of risk assessment models. J Natl Cancer Inst 2010; 102: 680-691.

31 Honrado E, Benitez J, Palacios J: The molecular pathology of hereditary breast cancer: genetic testing and therapeutic implications. Mod Pathol 2005; 18: 1305-1320.

32 STOET-VGKN. Dutch CGC referral criteria (Stichting Opsporing Erfelijke Tumoren Vereniging Klinische Genetica Nederland (STOET-VGKN) criteria), edition 1996 and 2001.

33 Schlich-Bakker KJ, ten Kroode HF, Warlam-Rodenhuis CC, van den Bout J, Ausems MG: Barriers to participating in genetic counseling and BRCA testing during primary treatment for breast cancer. Genet Med 2007; 9: 766-777.

34 Van Riel E, Warlam-Rodenhuis CC, Verhoef S, Rutgers EJ, Ausems MG: BRCA testing of breast cancer patients: medical specialists' referral patterns, knowledge and attitudes to genetic testing. Eur J Cancer Care (Engl) 2010; 19: 369-376.

35 Antoniou AC, Easton DF: Risk prediction models for familial breast cancer. Fut Oncol 2006; 2: 257-274.

36 Bouchardy C, Benhamou S, Fioretta G et al: Risk of second breast cancer according to estrogen receptor status and family history. Breast Cancer Res Treat 2011; 127: 233-241.

37 Warlam-Rodenhuis CC, Koot VC, van der Luijt RB, Vasen HF, Ausems MG: A prospective study on predictive factors linked to the presence of BRCA1 and BRCA2 mutations in breast cancer patients. Eur J Cancer 2005; 41: 1409-1415.

38 Lakhani SR, Van De Vijver MJ, Jacquemier J et al: The pathology of familial breast cancer: predictive value of immunohistochemical markers estrogen receptor, progesterone receptor, HER-2, and p53 in patients with mutations in BRCA1 and BRCA2. J Clin Oncol 2002; 20: 2310-2318.

39 Kwon JS, Gutierrez-Barrera AM, Young D et al: Expanding the criteria for BRCA mutation testing in breast cancer survivors. J Clin Oncol 2010; 28: 4214-4220.

40 Robertson L, Hanson H, Seal S et al: BRCA1 testing should be offered to individuals with triple-negative breast cancer diagnosed below 50 years. Br J Cancer 2012; 106: 1234-1238

41 Weitzel JN, Lagos VI, Cullinane CA et al: Limited family structure and BRCA gene mutation status in single cases of breast cancer. JAMA 2007; 297: 2587-2595.

42 National Institute for Health and Care Excellence. CG164 Familial breast cancer NICE guidance. Available at:. http://guidance.nice.org.uk/CG164/NICEGuidance/pdf/ English 2013 (last accessed on 26 June 2014).

cc)(i)(2) (2) This work is licensed under a Creative Commons Attribution-NonCommercial-ShareAlike 3.0 Unported License. The images or other third party material in this article are included in the article's Creative Commons license, unless indicated otherwise in the credit line; if the material is not included under the Creative Commons license, users will need to obtain permission from the license holder to reproduce the material. To view a copy of this license, visit http://creativecommons.org/ licenses/by-nc-sa/3.0/

Supplementary Information accompanies this paper on European Journal of Human Genetics website (http://www.nature.com/ejhg) 